\title{
Exponential or Pareto: towards an evolution of equal opportunity
}

Author: Yong Tao

Affiliation: College of Economics and Management, Southwest University, Chongqing, China

Acknowledgement: This work was supported by the Social Science Planning Project of Chongqing (Grant No. 2019PY40).

Author Contributions: Yong Tao performed the research in this paper independently.

Author Information: The author declares no competing interests. Correspondence and requests for materials should be addressed to Yong Tao (taoyingyong@yahoo.com). 
Pareto distribution and exponential distribution are related by the generalized Pareto distribution ${ }^{1-3}$, which has been proposed to describe the income structure of the total population ${ }^{4-5}$. The underlying mechanism for driving the Pareto distribution has been known as the Matthew effect of income accumulation ${ }^{6-10}$. Today, the Pareto distribution has been observed universally in the richest class ${ }^{11-}$ 14 (1\% 3\% of populations); however, this distribution could dominate a larger proportion of populations when the investigation dated back to Renaissance Europe $^{15}$, Hungarian medieval society ${ }^{16}$, and ancient Egypt ${ }^{17}$. By contrast, the underlying mechanism for driving exponential income distribution is due to the equal opportunity of market competition ${ }^{18-20}$, which radically differs from the Matthew effect. Here, we empirically find that, during the last 40 years, the income structures of different market-economy countries uniformly exhibit a two-class pattern, in which the great majority of populations obeys an exponential distribution and only the remaining (richest) part follows a Pareto distribution. In particular, we empirically show how the income structure in China evolved to an exponential distribution after the market-oriented economic reformation. The finding of a larger proportion of populations evolving to an exponential income distribution may reveal a potential trend of human civilization towards equal opportunity.

Through time, roughly speaking, we humans have explored four social structures, from hunter-gatherer societies, slave-based societies, feudal hierarchical societies, to various types and levels of democratic societies. At the early stage of human society, humans' behaviors were dominated by "The law of the jungle", which causes the Matthew effect in material allocations. Nowadays, it has been known that the Matthew effect leads to the Pareto distribution of income $e^{6-10}$. At the end of the 19th century, the economist Vilfredo Pareto discovered that ${ }^{21}$ the rich part of populations in several European countries, kingdoms and cities for the 15th-19th centuries obeys a Pareto distribution. Later, Pareto's finding was confirmed by investigating market-economy countries in the 20th and 21th centuries ${ }^{11-14,22}$. In particular, there had been evidence showing that 
the income structure of an ancient Egypt city in the 14th century BC followed a Pareto distribution ${ }^{17}$. Because slaves had no property right of personal income, this evidence implies that slave-based societies might undergo a Pareto distribution of the total population. As the time came to the end of the 20th century, it had been found that the income structures of market-economy countries around the world uniformly exhibited a two-class pattern 19-20, 23-33: the majority of populations obeyed an exponential distribution and only the remaining (richest) part followed a Pareto distribution. Empirical evidence had covered more than 66 countries $^{19}$, ranging from Europe to Latin America, North America, and Asia. Recently, Blanchet et $\mathrm{al}^{4-5}$ proposed to use the generalized Pareto distribution to describe the income structure of the total population, where the generalized Pareto distribution is defined as follows ${ }^{1-3}$ :

$$
F_{\xi}(t \geq x)=\left(1+\xi \frac{x-\mu}{\theta}\right)^{-1 / \xi}
$$

where $\xi>0, x$ denotes the income level, and $F_{\xi}(t \geq x)$ denotes the fraction of population with the income higher than $x$.

The generalized Pareto distribution is a fairly general family which includes the Pareto distribution and the exponential distribution as two special cases ${ }^{1-3}$. To see this, by ordering $\mu=\theta / \xi$, equation (1) yields the Pareto distribution:

$$
F_{\xi}(t \geq x)=(x / \mu)^{-1 / \xi},
$$

where the Pareto exponent is denoted by $1 / \xi$, which measures the degree of income inequality ${ }^{14}$, that is, a larger Pareto exponent is associated with a lower income inequality. Intuitively, a lower income inequality should correspond to a more equalopportunity society. Therefore, we anticipate that, as the Pareto exponent $1 / \xi \rightarrow \infty$ (or $\xi \rightarrow 0$ ), equation (1) yields a distribution being close to equal opportunity:

$$
F_{0}(t \geq x)=\lim _{\xi \rightarrow 0}\left(1+\xi \frac{x-\mu}{\theta}\right)^{-1 / \xi}=e^{-\frac{x-\mu}{\theta}},
$$

which is an exponential distribution.

Theoretically, it has been shown that ${ }^{18-20}$ the exponential income distribution (3) emerges spontaneously in an idealized market economy with equal-opportunity, which is a decentralized self-organizing economic network, just as described by the Arrow- 
Debreu general equilibrium model ${ }^{34}$. Here, we briefly introduce such an economic network. To this end, we consider an "N-agent non-cooperative game", where are $N$ agents, each of whom operates a firm, and each agent's status is equal. In this economic network, all of these firms pursue maximum profit, and all of these agents exchange with each other to obtain maximum satisfaction. Arrow and Debreu prove that ${ }^{34}$, for each agent, if there is free of cost for information transmission and transaction, then all of these agents will reach consensuses on resource allocation (so-called the "general equilibrium"). Tao further proves that ${ }^{18,35}$, if the property right of each firm is endowed to a different agent, then these consensuses lead to the exponential income distribution (3). However, information transmission and transaction would take additional cost. Therefore, neoclassical economists" ${ }^{36}$ have to assume an imaginary "Walrasian auctioneer", who has an advanced technology to eliminate the cost for information transmission and transaction, to selflessly assist all agents in reaching exchanges without any transaction cost. Unfortunately, when an agent has the potential to become a Walrasian auctioneer, motivated by selfishness it may instead take advantage of technology to acquire more incomes, undermining equal opportunity of market competition. For example, the internet business platforms (such as Amazon and Alibaba) could have the potential to become Walrasian auctioneers; however, all of them eventually become oligopolies benefiting from the Matthew effect. This explains why the Pareto distribution is observed in the top income class in a market economy. Although the internet technology did not eliminate beneficiaries of the Matthew effect, with rise of information technology, the block-chain is further proposed to play the role of a Walrasian auctioneer. This implies that there seems to be an intrinsic motivation ${ }^{20}$ for driving a human society to achieve an equal-opportunity economic network. Therefore, it may be conjectured that a larger proportion of populations in a marketeconomy country is evolving towards to an exponential income distribution.

Here, we employ the household data from four market-economy countries to verify that a larger proportion of populations is evolving towards the exponential income distribution (3). The four countries include three typical developed economy (United Kingdom, United States, and Canada) and one typical developing economy (China). In 
particular, because the China is a special sample that has undergone the transition from a planned economy to a market economy, it is more interesting to check if there is a transition towards an exponential income distribution after the market-oriented economic reformation. We first demonstrate that it is more reasonable to analyze the household income data by using the cumulative distribution function (rather than the density distribution function). In particular, when one employs the density distribution function to analyze the household income data, the results may be inconsistent. In Figure 1, we use the household income data from the United Kingdom in 2010 to show this inconsistency: The shape of the density distribution of income may depend on the bin width of the histogram. For example, when the bin width is denoted by 4000 GBP, Figure 1a shows a right-skewed density distribution with a peak, which is close to a Log-normal distribution or a Gamma distribution. Unfortunately, when the bin width is increased to $8000 \mathrm{GBP}$, as shown in Figure 1b, the shape of density distribution begins to deviate from a Log-normal distribution or a Gamma distribution. In particular, when the bin width is increased to $12000 \mathrm{GBP}$, the shape of density distribution turns to a monotonic function, see Figure 1c. This means that, for the same data, density distribution may lead to inconsistent graphs. However, when one uses the cumulative distribution function to describe the household income data, there are no such inconsistencies. Figure 1d shows that the great majority of populations in the United Kingdom obeys the exponential income distribution (3), which is a cumulative distribution function. Henceforth, we always employ the cumulative distribution function to analyze the household income data.

\section{[Insert Figure 1 here]}

Furthermore, Figure 2 shows that the income structures of China (in 2015), United Kingdom (in 2017), Canada (in 2018) uniformly exhibit a two-class pattern in which the great majority of populations obeys the exponential income distribution (3) and only the remaining (richest) part follows a Pareto distribution. Figure 2 also shows that the great majority of populations in the United States (in 2019) follows the exponential 
income distribution (3). The dataset is gathered from the most recent years. The empirical observation supports our conjecture that a larger proportion of populations in a market-economy country is evolving towards to an exponential income distribution.

\section{[Insert Figure 2 here]}

Here, we particularly employ the household income data in China from 1978 to 2000 to demonstrate that the exponential income distribution (3) emerges spontaneously in a market economy, as argued by Tao ${ }^{18}$. This period of time covers the main stages of China's market-oriented economic reformation. In Figure 3, we empirically show that the income structure in China gradually evolved to an exact exponential distribution after the 1990s. By contrast, the income structure in China around the 1990s only approximately obeyed the exponential distribution, while the income structures in 1978 and 1980 radically differed from the exponential distribution. This is strong evidence that the exponential income distribution (3) emerges spontaneously in a market economy.

\section{[Insert Figure 3 here]}

The evolution of human society seems to reveal a trend from hunter-gatherer societies, slave-based societies, feudal hierarchical societies, to various types and levels of democratic societies. Along with historical advancement, human societies are getting close to an equal-opportunity economic network. This is reflected as that a larger proportion of populations is evolving towards to an exponential income distribution, while the Pareto distribution is squeezed into a fairly small proportion. By contrast, the Pareto distribution dominated a larger proportion of populations in the period of advocating the law of the jungle (e.g., slave-based societies), which causes the Matthew effect in material allocations. Today, with the continuous rise of digital interconnection, instantaneous communications, media, and blog-based echo chambers, our societies have been promoted to a more democratic and equal-opportunity structure. This 
structure may be further improved by the rise of artificial intelligence and block-chain technology. 


\section{Reference:}

1. Singh, S. K. and Maddala, G. S. A function for the size distribution of income. Econometrica 44, 963-970 (1976)

2. Cowell, F. A., Measuring Inequality. LSE Economic Series. Oxford University Press. (2000)

3. Jenkins, S. P. Pareto Models, Top Incomes and Recent Trends in UK Income Inequality. Economica 84, 261-289 (2016)

4. Blanchet, T., Fournier, J. and Piketty, T. Generalized Pareto Curves: Theory and Applications. Working Papers 201703, World Inequality Lab. (2017)

5. Blanchet, T., Garbinti, B., Goupille-Lebret, J., and Martínez-Toledano. C. Applying Generalized Pareto Curves to Inequality Analysis. AEA Papers and Proceedings 108, 114-118 (2018)

6. Champernowne, D. G. A Model of Income Distribution. Economic Journal 63, 318-351 (1953)

7. Wold, H. O. A. and Whittle, P. A Model Explaining the Pareto Distribution of Wealth. Econometrica, 25, 591-595 (1957)

8. Angle, J. Deriving the Size Distribution of Personal Wealth from "The Rich Get Richer, the Poor Get Poorer". Journal of Mathematical Sociology 18, 27-46 (1993)

9. Benhabib, J., Bisin, A. and Zhu, S. The Distribution of Wealth and Fiscal Policy in Economies With Finitely Lived Agents. Econometrica, 79, 123-157 (2011)

10. Tao, Y. Universal Laws of Human Society's Income Distribution, Physica A 435, 89-94 (2015)

11. Mandelbrot, B. The Pareto-Levy law and the distribution of income. International Economic Review 1, 79-106 (1960)

12. Atkinson, A. B., Piketty, T., and Saez, E. Top Incomes in the Long Run of History. Journal of Economic Literature 49, 3-71 (2011),

13. Aoki, S. and Nirei, M. Zipf's Law, Pareto's Law, and the Evolution of Top Incomes in the United States. American Economic Journal: Macroeconomics, 9, 36-71 (2017) 
14. Jones, C. I. and Kim, J. A Schumpeterian Model of Top Income Inequality. Journal of Political Economy 126, 1785-1826 (2018)

15. Souma, W. Empirical Science of Financial Fluctuations: The Advent of Econophysics, Springer, Tokyo. (2002)

16. Hegyi, G., Neda, Z., and Santos, M. A. Wealth distribution and Pareto's law in the Hungarian medieval society. Physica A 380, 271-277 (2007)

17. Abul-Magd, A. Y. Wealth distribution in an ancient Egyptian society. Physical Review E 66, 057104 (2002)

18. Tao, Y. Spontaneous economic order, Journal of Evolutionary Economics 26, 467-500 (2016)

19. Tao, Y., Wu, X., Zhou, T., Yan, W., Huang, Y., Yu, H., Mondal, B., and Yakovenko, V. M. Exponential structure of income inequality: evidence from 67 countries. Journal of Economic Interaction and Coordination 14, 345-376 (2019)

20. Tao, Y., Sornette, D., and Lin, L. Emerging social brain: a collective selfmotivated Boltzmann machine. Chaos, Solitons \& Fractals 143, 110543 (2021)

21. Pareto, V. Cours d'Economie Politique. L' Universite de Lausanne. (1897)

22. Aoyama, H., Souma,W., Nagahara, Y., Okazaki, M. P., Takayasu, H., and Takayasu, M. Pareto's law for income of individuals and debt of bankrupt companies. Fractals 8, 293-3000 (2000)

23. Dragulescu, A. and Yakovenko, V. M. Exponential and power-law probability distributions of wealth and income in the United Kingdom and the United States. Physica A 299, 213-221 (2001)

24. Nirei, M. and Souma, W. A Two Factor Model of Income Distribution Dynamics. Review of Income and Wealth 53, 440-459 (2007)

25. Newby, M., Behr, A., and Feizabadi, M. S. Investigating the distribution of personal income obtained from the recent U.S. data. Economic Modelling 28, 1170$1173(2011)$

26. Prinz, A. Do capitalistic institutions breed billionaires? Empirical Economics $51,1319-1332(2016)$ 
27. Oancea, B., Andrei, T., and Pirjol, D. Income inequality in Romania: The exponential-Pareto distribution. Physica A 469, 486-498 (2017)

28. Shaikh, A. Income distribution, econophysics and Piketty. Review of Political Economy 29, 18-29 (2017)

29. Irwin. R. J. and Irwin, T. C. Stability of an exponential distribution for New Zealand taxable personal income. New Zealand Economic Papers 51, 49-59 (2017)

30. Rosser, J. B. The Minsky moment as the revenge of entropy. Macroeconomic Dynamics 24, 7-23 (2019)

31. Ma, L. and Ruzic, D. Globalization and top income shares. Journal of International Economics 125, 103312 (2020)

32. Lim, G. and Min, S. Analysis of Solidarity Effect for Entropy, Pareto, and Gini Indices on Two-Class Society Using Kinetic Wealth Exchange Model. Entropy 22, 386 (2020)

33. Diaz, J. D., Cubillos, P. G., and Grinen, P. T. The exponential Pareto model with hidden income processes: Evidence from Chile. Physica A 561, 125196 (2021)

34. Arrow, K. J. and Debreu, G. Existence of an Equilibrium for a competitive economy. Econometrica 22, 265-290 (1954)

35. Tao, Y. Exponential Income Distribution and General Equilibrium Growth: A Survey for the United Kingdom. Available at SSRN: https://ssrn.com/abstract=3411095

36. Varian, H. R. Microeconomic analysis, 3rd edn. Norton \& Company, Inc., New York (1992) 


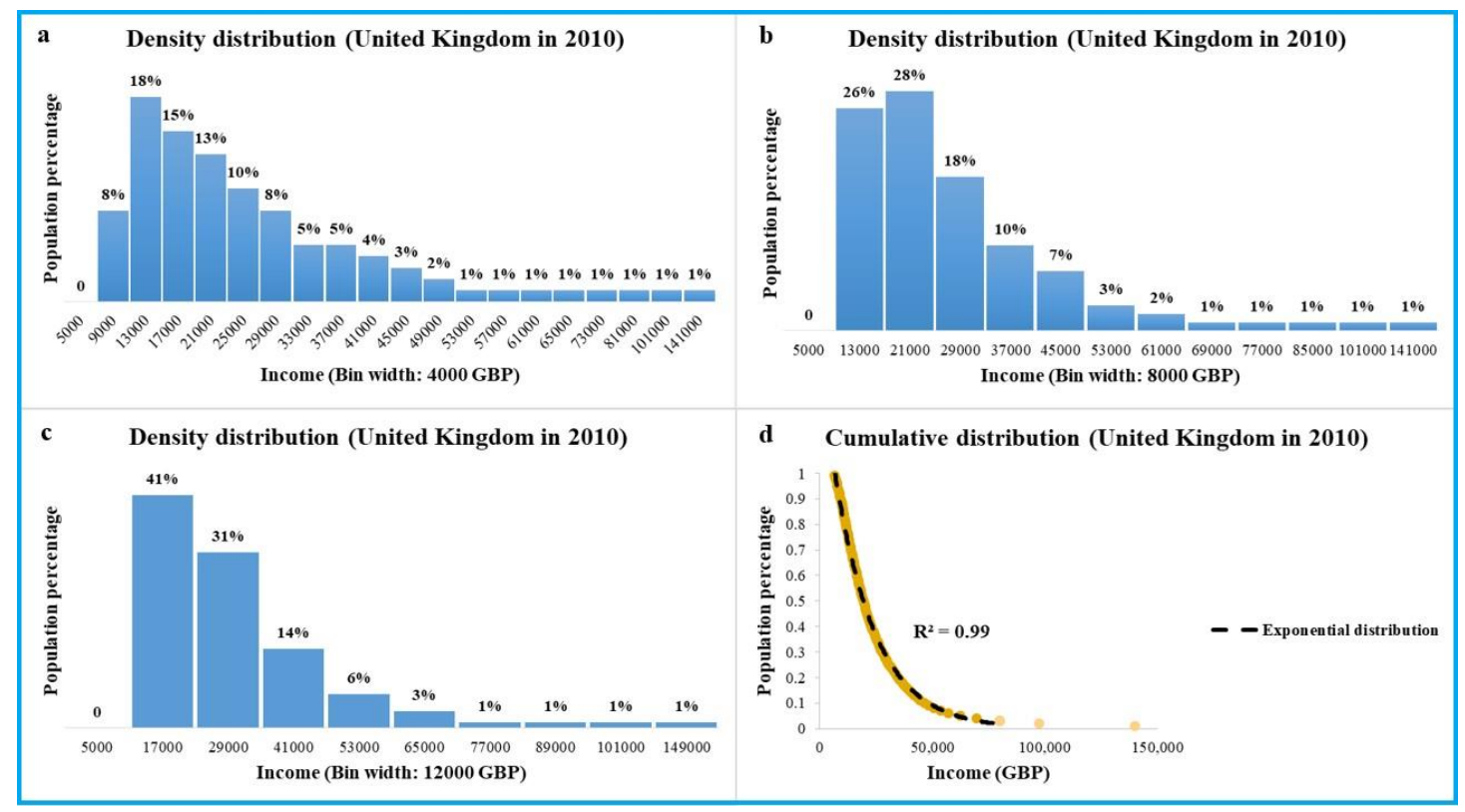

Figure 1. | Density distribution may lead to inconsistent graphs ${ }^{1}$. a: When the bin width is denoted by $4000 \mathrm{GBP}$, the histogram of density distribution is a right-skewed graph with a peak, which is close to a Log-normal distribution or a Gamma distribution. b: When the bin width is increased to $8000 \mathrm{GBP}$, the histogram of density distribution begins to deviate from a Log-normal distribution or a Gamma distribution. c: When the bin width is increased to $12000 \mathrm{GBP}$, the histogram of density distribution turns to a monotonic function. $\mathbf{d}$ : The cumulative distribution of income exhibits an exponential function.

\footnotetext{
${ }^{1}$ Data resource: https://www.gov.uk/government/statistics/percentile-points-from-1-to-99-fortotal-income-before-and-after-tax
} 


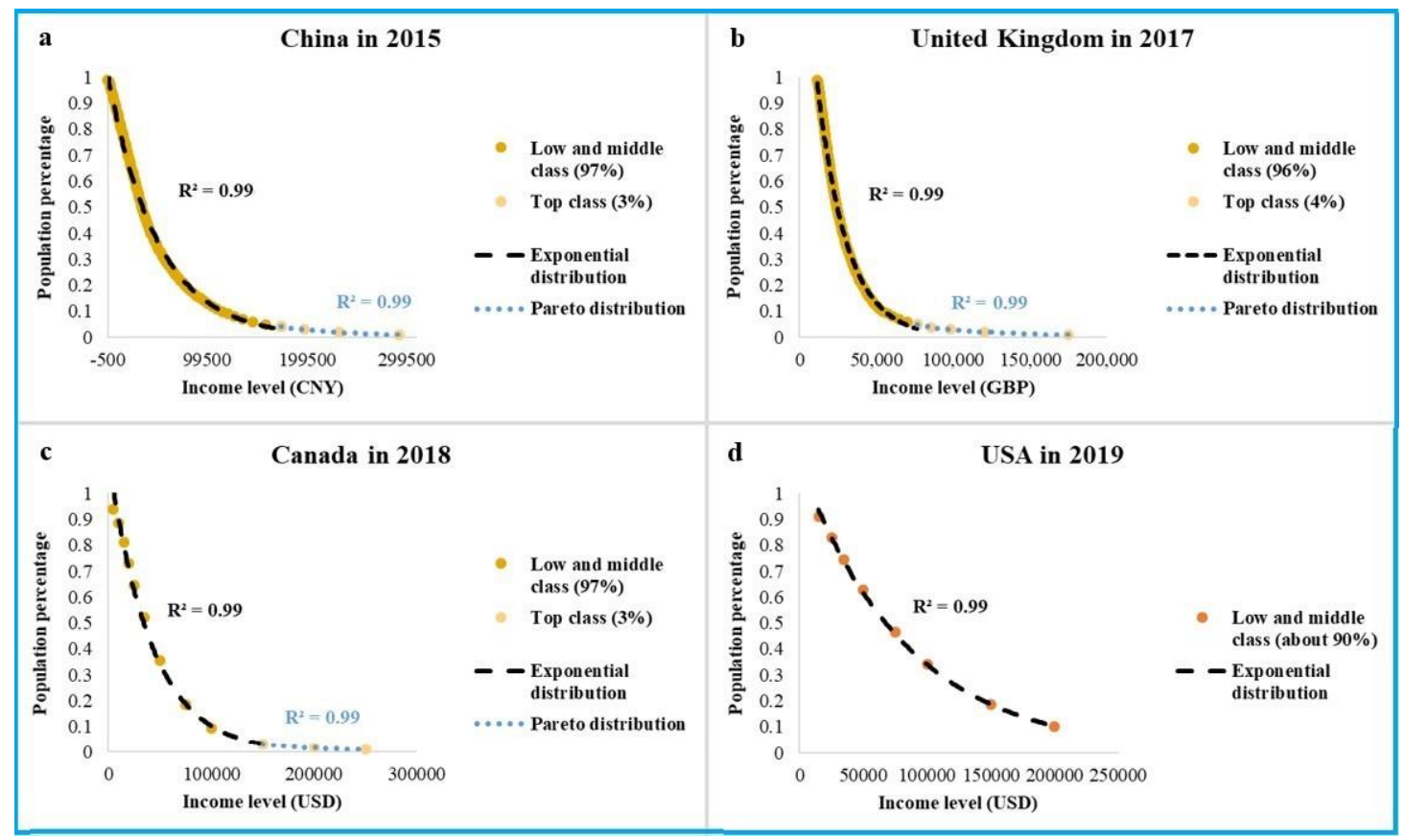

Figure 2. | A larger proportion of populations evolving towards to an exponential income distribution. a: The low and middle income classes (97\% of populations) in China obeys an exponential income distribution ${ }^{2}$. b: The low and middle income classes (96\% of populations) in the United Kingdom obeys an exponential income distribution ${ }^{3}$. c: The low and middle income classes (97\% of populations) in Canada obeys an exponential income distribution ${ }^{4}$. d: The low and middle income classes (more than $90 \%$ of populations) in the United States obeys an exponential income distribution ${ }^{5}$.

\footnotetext{
${ }^{2}$ Data resource for China: http://wid.world/data/

${ }_{3}^{3}$ Data resource for the United Kingdom: https://www.gov.uk/government/statistics/percentilepoints-from-1-to-99-for-total-income-before-and-after-tax

${ }^{4}$ Data resource for Canada: https://www150.statcan.gc.ca/t1/tbl1/en/tv.action?pid=1110000801

${ }_{5}^{5}$ Data resource for the United States: https://www.statista.com/statistics/203183/percentagedistribution-of-household-income-in-the-us/
} 


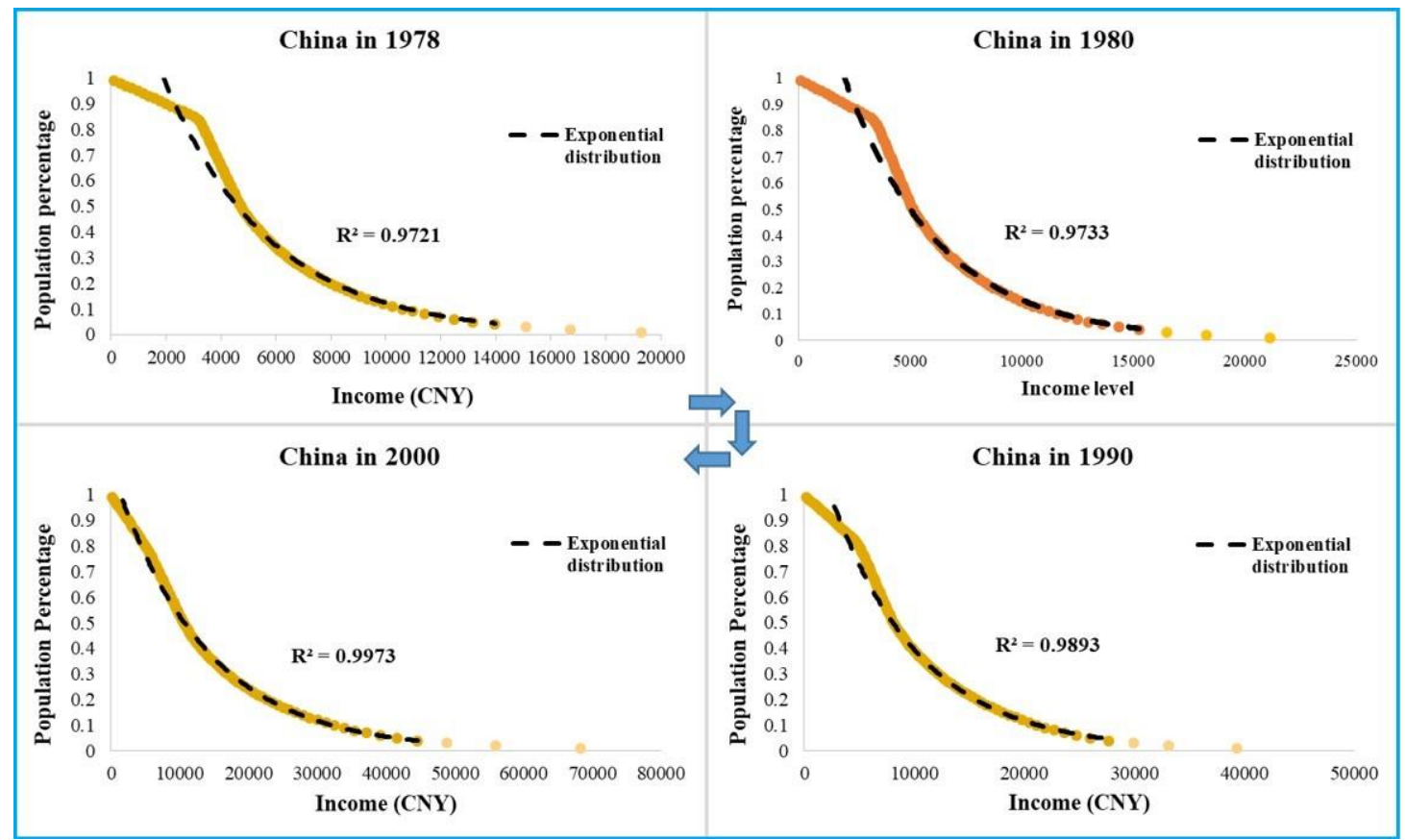

Figure 3. | The income structure in China gradually evolved to an exponential distribution after the market-oriented economic reformation ${ }^{6}$.

${ }^{6}$ Data resource for China: http://wid.world/data/ 